\title{
Simulation of ultrasound backscatter images from fish
}

Pham, An Hoai; Stage, Bjarne; Hemmsen, Martin Christian; Lundgren, Bo; Pedersen, Mads Møller; Pedersen, Tina Bock; Jensen, Jørgen Arendt

Published in:

SPIE Medical Imaging 2011

Link to article, DOI:

$10.1117 / 12.878012$

Publication date:

2011

Document Version

Publisher's PDF, also known as Version of record

Link back to DTU Orbit

Citation (APA):

Pham, A. H., Stage, B., Hemmsen, M. C., Lundgren, B., Pedersen, M. M., Pedersen, T. B., \& Jensen, J. A. (2011). Simulation of ultrasound backscatter images from fish. In SPIE Medical Imaging 2011: Physics of Medical Imaging (pp. Article Number: 796152). SPIE - International Society for Optical Engineering. Proceedings of SPIE - The International Society for Optical Engineering https://doi.org/10.1117/12.878012

\section{General rights}

Copyright and moral rights for the publications made accessible in the public portal are retained by the authors and/or other copyright owners and it is a condition of accessing publications that users recognise and abide by the legal requirements associated with these rights.

- Users may download and print one copy of any publication from the public portal for the purpose of private study or research.

- You may not further distribute the material or use it for any profit-making activity or commercial gain

- You may freely distribute the URL identifying the publication in the public portal 


\title{
Simulation of ultrasound backscatter images from fish
}

\author{
An Hoai Pham ${ }^{a, b}$, Bjarne Stage ${ }^{a}$, Martin Christian Hemmsen ${ }^{b, d}$, Bo Lundgren ${ }^{a}$, Mads Møller \\ Pedersen ${ }^{b, c}$, Tina Bock Pedersen ${ }^{c}$ and Jørgen Arendt Jensen ${ }^{b}$. \\ ${ }^{a}$ National Institute of Aquatic Resources, Technical University of Denmark, \\ Jægersborg Allé 1, 2920 Charlottenlund, Denmark; \\ ${ }^{b}$ Center for Fast Ultrasound Imaging, Dept. of Elec. Eng. Build. 349, Technical University of \\ Denmark, DK-2800 Kgs. Lyngby, Denmark; \\ ${ }^{c}$ Department of Radiology, Section of Ultrasound, Rigshospitalet, Copenhagen, Denmark; \\ ${ }^{d}$ BK Medical, Mileparken 34, DK-2730 Herlev, Denmark
}

\begin{abstract}
The objective of this work is to investigate ultrasound (US) backscatter in the MHz range from fish to develop a realistic and reliable simulation model. The long term objective of the work is to develop the needed signal processing for fish species differentiation using US. In in-vitro experiments, a cod (Gadus morhua) was scanned with both a BK Medical ProFocus 2202 ultrasound scanner and a Toshiba Aquilion ONE computed tomography (CT) scanner. The US images of the fish were compared with US images created using the ultrasound simulation program Field II. The center frequency of the transducer is $10 \mathrm{MHz}$ and the Full Width at Half Maximum (FWHM) at the focus point is $0.54 \mathrm{~mm}$ in the lateral direction. The transducer model in Field II was calibrated using a wire phantom to validate the simulated point spread function. The inputs to the simulation were the CT image data of the fish converted to simulated scatter maps. The positions of the point scatterers were assumed to be uniformly distributed. The scatter amplitudes were generated with a new method based on the segmented CT data in Hounsfield Units and backscatter data for the different types of tissues from the literature. The simulated US images reproduce most of the important characteristics of the measured US image.
\end{abstract}

Keywords: ultrasound, simulation, acoustical properties, small animal experiment, CT

\section{INTRODUCTION}

The management of fisheries of commercially important pelagic fish stocks relies on abundance estimates provided by data from scientific surveys. ${ }^{1}$ Abundance estimates of pelagic fish species are commonly obtained by acoustic survey techniques. ${ }^{2}$ During an acoustic survey of a sea area the acoustic energy scattered from layers in the pelagic volume is measured accurately along transects. A mean target strength for the fish population is obtained for each of a number of subareas by using knowledge about the size and species distribution and expected target strengths of the fish present. The total energy scattered from fish in the subarea as estimated from the measurements divided by the mean target strength then gives the fish population size in the subarea. Each species is then allocated a portion again based on the species and size distributions. Knowledge about the species present and their size distributions is obtained by physically sampling the pelagic volume using methods such as trawling. Trawling is a costly, time-consuming, and under certain circumstances imprecise method for acquiring knowledge about local species and size composition. An alternative could be an acoustic method for in-situ identification of fish species and estimation of size distribution.

Acoustic surveys are traditionally conducted with hull mounted transducers. As the acoustic attenuation increases with frequency, the highest usable frequency is determined by the water depth. Typical frequencies used for acoustic surveys are in the range $18-200 \mathrm{kHz}$. Some success has been achieved in identification of fish species using a multiple frequency approach in the $\mathrm{kHz}$ range, ${ }^{3}$ and the method is currently utilized as a supplement to physical sampling. Further improvement in identification accuracy is expected when using high frequency, wide band acoustics. Due to the limited range of high frequency acoustics, such transducers must be mounted on remotely operated vehicles or towed bodies in order to bring the transducer in the vicinity of the fish for measurement. Experimental determination of the target strength of fish is an elaborate procedure, ${ }^{4}$ as the target strength must be measured for each species, with individuals of different size, with multiple frequencies and many different angles of incidence. The experimental procedure can be supplemented by modeling of the acoustic scattering from fish. Not only can modeling confirm and extend the experimental results, but it can also provide valuable insight into which parts of the fish dominate the scattering process. In the $\mathrm{kHz}$ range, the acoustic 
wavelength is typically much larger than the fish, and traditional low frequency scattering models have been employed. ${ }^{5}$ In the $\mathrm{MHz}$ region of frequencies the wavelength becomes much smaller than the fish and other modeling approaches must be taken. Medical ultrasound utilizes acoustics in the $\mathrm{MHz}$ range, so it seems natural to utilize the modeling methods employed in this field ${ }^{6}$.

The subject of this paper is modeling of acoustic backscattering from fish in the MHz region. The modeling is verified by comparison with experimentally measured backscattering from fish samples.

\section{OVERVIEW OF THE METHOD}

The experiments in this work were performed in-vitro using a cod (Gadus morhua), which was scanned with both an ultrasound scanner and a CT scanner. The cod was not alive in this experiment, and the time difference between the two measurements was 3 hours. The block diagram of the experiment is presented in Fig.1. The degree of resemblance between measured and simulated US images was determined by visual inspection. The procedure of the experiment is illustrated in the figure. It consists of the following steps: preparation of fish sample and adjustment of instrument parameters; acquisition of ultrasound (US) and CT data; and finally simulation of US images based on the CT data. The following sections will describe the procedure in detail.

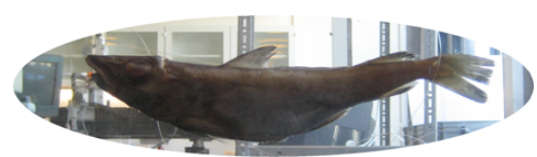

Fish sample

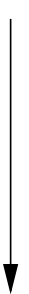
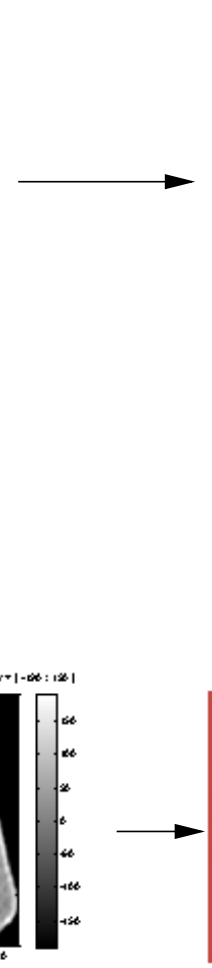

CT scanning

CT Image

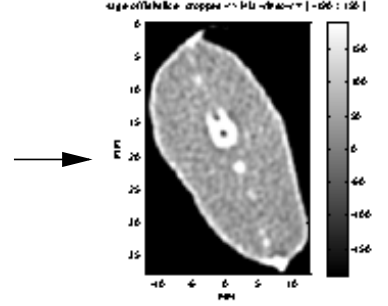

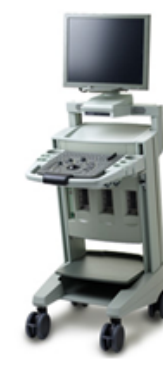

BK Medical Ultrasound scanner

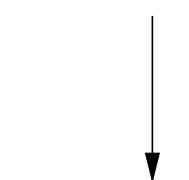

Figure 1. Block diagram of the in-vitro experiment.

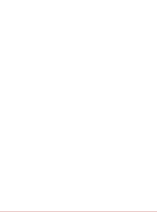

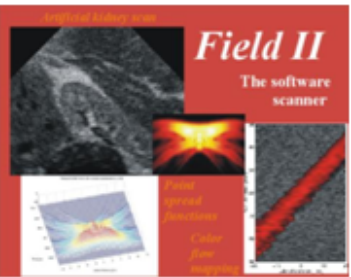

Field II program

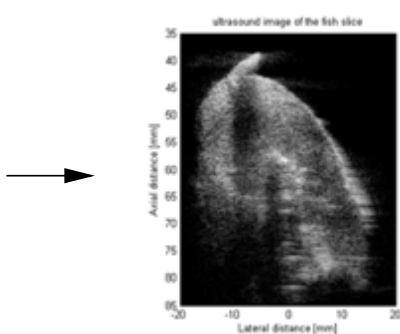

Measured US image
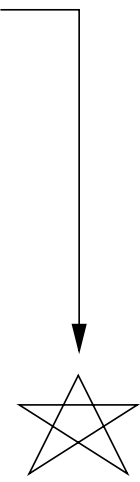

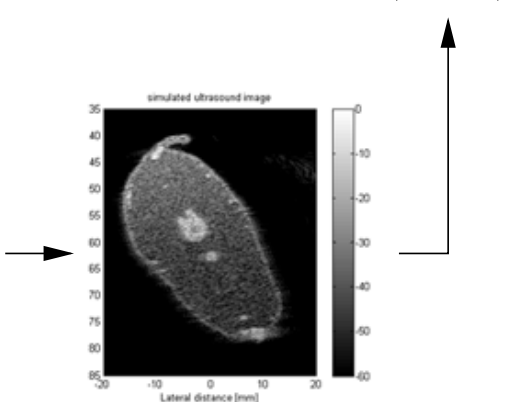

Simulated US image

\section{EXPERIMENT}

The cod was suspended in a fixture by fishing lines during the US scan as well as the CT scan. In addition, the fish and the fixture were encased in a box during the CT scan to avoid contamination of the hospital environment where the CT scanner used was localized. One of the challenges of this experiment was to choose the material for the fixture and the box to avoid noise and artifacts in the CT images of the fish. Based on the literature, Polyvinyl chloride (PVC) was chosen as a suitable material for fixtures as well as boxes. A pilot CT experiment using a banana instead of a fish was performed 
before the actual experiment to test fixture and box and to adjust the parameters of the CT scanner. The banana was chosen as test specimen as its X-ray properties resemble the soft tissue parts of fish. The result shown in Fig. 2 indicated that the influence of the PVC fixture and box on the CT images of the banana was negligible. An additional CT scan was made using the same setup but with the addition of one liter of water in the box. The addition of water reduced the contrast and added noise to the images. Based on this result it was decided to perform the CT scan of the cod without water in the box.

a)

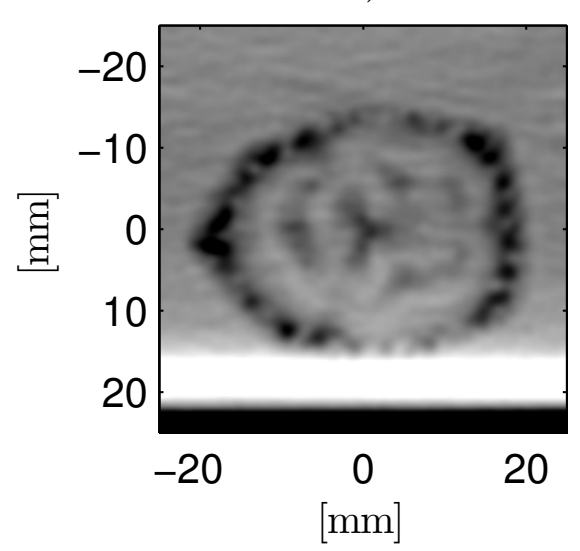

b)

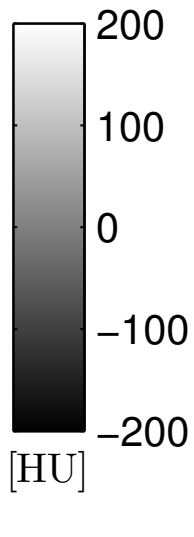

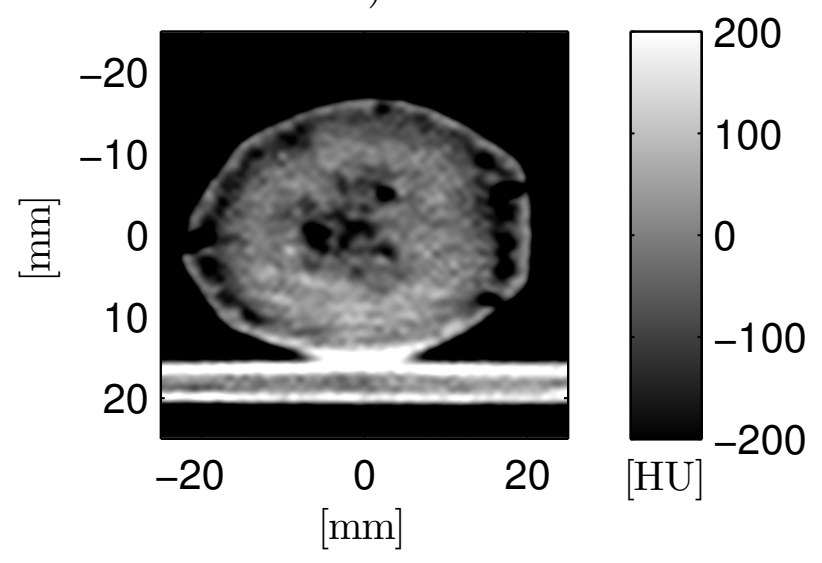

Figure 2. CT images of banana, with water (a), without water (b). The two CT images are not comparable. They are reconstructed with different pixel sizes.

\subsection{Acoustic scanning}

The acoustic scanning was done at the Center for Fast Ultrasound Imaging (CFU). The fish used was a cod with a length of approximately $210 \mathrm{~mm}$. It was suspended using $0.1 \mathrm{~mm}$ fishing lines in a PVC fixture and placed in a small aquarium container filled with degassed water. The scanning was performed using a BK Medical Profocus 2202 scanner, equipped with a dedicated research interface connected to a PC through a X64-CL Express camera_link interface (Dalsa, Waterloo, Ontario, Canada). The system allows the acquisition of digitally beamformed RF echo data. ${ }^{8}$ A BK Medical 880410 $\mathrm{MHz}$ linear array transducer was attached to an electro-mechanical linear scanner on top of the aquarium. The transducer head was approximately $30 \mathrm{~mm}$ below the water surface. The transmit focus point of the transducer was at a range of $45 \mathrm{~mm}$, and dynamic focusing was used in receive mode with the F\# equal to 0.5. The overall gain and the time gain compensation (TGC) were adjusted, so that the full speckle pattern could be seen clearly in the soft tissue areas in the US images. Digitally beam-formed RF echo data and a parameter setup file of the scanner were retrieved using the program CFU Grabber. ${ }^{8}$ The measured US images of the fish were generated from the RF data using MATLAB. The parameter files were saved to be used afterwards for the simulations. The typical scan-time to cover the $200 \mathrm{~mm}$ fish from nose to tail was about 1 minute.

The same US scanner setup was used to obtain US images of a wire phantom with 8 metal wires. This data is necessary to verify the settings of the simulation program in the simulation stage.

\subsection{CT scanning}

The CT scanning of the fish was done at Rigshospitalet in Copenhagen. Immediately after the US scanning of the wire phantom, the fish and the fixture were put into the PVC box without water, and the sealed box was transported to the hospital. The box with the fish was scanned with a Toshiba Aquilion ONE CT scanner. It can produce CT images with a slice thickness of $0.5 \mathrm{~mm}$ and pixel sizes of $0.274 \mathrm{~mm} \times 0.274 \mathrm{~mm}$. The CT data, given in Hounsfield Units, were stored onto a harddrive, and the CT images of the fish were generated using MATLAB. In addition the CT data were used to generate "3D images" of the fish. By selecting suitable threshold levels, a 3D overview of anatomical details such as the bone structure of the fish can be created. 


\section{SIMULATION}

The Field $\mathrm{II}^{6,7}$ software was used to generate the simulated US images. This program uses linear acoustics to simulate the transmitted, backscattered, and received US fields. The simulation of the final US images of the fish was prepared in two stages: First the wire phantom was simulated and compared to the output of the US-scan image to verify the parameter setting (calibration) of the transducer model for the Field II; then US scatterer maps were generated from the CT images of the fish.

\subsection{Simulation of the wire phantom}

The inputs used for the simulation of the wire phantom with Field II were the transducer model and the positions of the point scatterers representing a model of the wire phantom. The transducer model and the medium parameters were set according to the information in the parameter files that were saved during the acoustic scanning. The positions of the scatterers were found by locating the scattering maximum points in the US images of the wire phantom. An example of a measured US image and the corresponding simulated US image of the wire phantom are presented in Fig. 3

a)

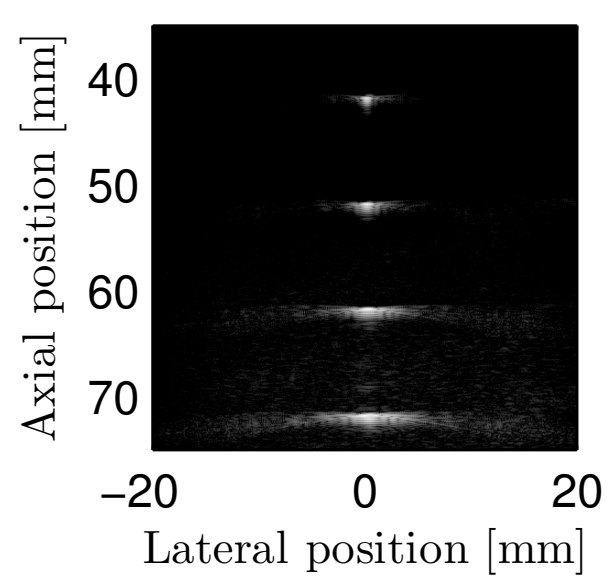

b)

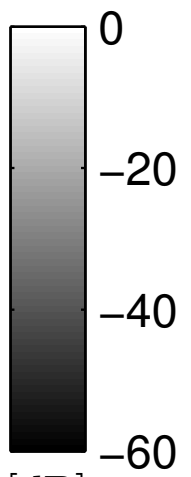

$[\mathrm{dB}]$

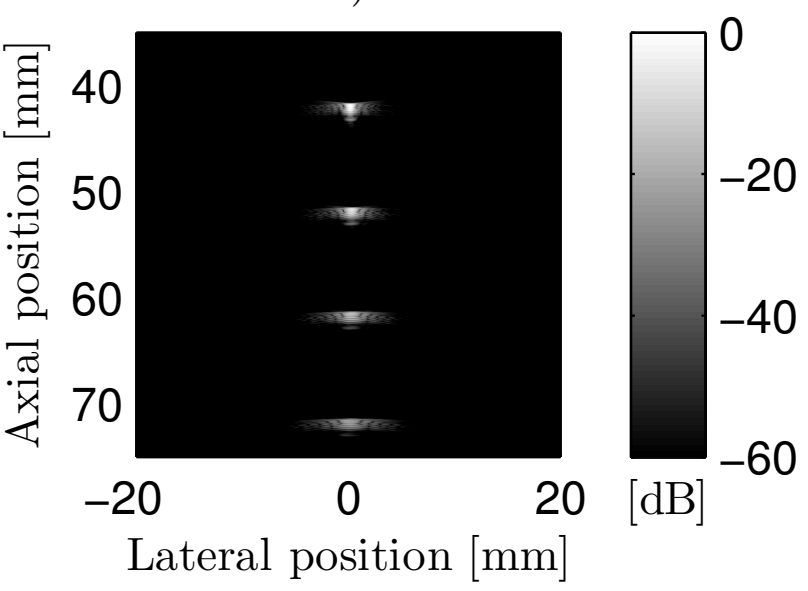

Figure 3. Measured US image of the wire phantom (a), Simulated US image of the wire phantom (b). The scales show the lateral distance from the acoustic centerline and the vertical distance from the transducer surface. Gray level indicates the relative signal strength in $\mathrm{dB}$.

The simulated Full Width at Half Maximum (FWHM) values at the transmit focus point of the US imaging system used in this work were $0.54 \mathrm{~mm}$ and $0.3 \mathrm{~mm}$ in the lateral and axial directions, respectively. The positions of the point scatterers were $41.8 \mathrm{~mm}, 51.7 \mathrm{~mm}, 61.6 \mathrm{~mm}$, and $71.6 \mathrm{~mm}$ away from the transducer in the axial direction. With the chosen parameters the discrepancy between the FWHM of the measured and simulated resolution were $6.7 \%, 0.6 \%, 0.1 \%$ and $10.4 \%$ in the lateral direction and $17 \%, 15 \%, 16.7 \%$, and $21 \%$ in the axial direction, respectively. Pulse echo waves from a plane parallel to the surface of the US transducer was measured and used as the excitation wave for the simulation.

\subsection{Simulation of the fish}

The procedure for simulation of the fish was similar to the simulation of the wire phantom. The same transducer model and the same medium parameter were used. But the scatterers had to be modeled using a more elaborate method.

Different methods have been used to convert CT data into a US scatterer distribution. In the study of Dillenseger et al., ${ }^{9}$ the scatterers were distributed using the 1D marked regularity model and a fast Hilbert filling curves algorithm. Their CT images were segmented into air, fat, soft tissue and bone. The regions were assigned the fixed tissue acoustical impedances that have been found in the literature. The resulting simulated images could not capture the shadowing effect of the real US images. Another approach used the combination of reflection and transmission images as in the case of Wein et al. study. ${ }^{10}$ The statistical similarity metrics like Mutual Information and Correlation Ratio were used to assess the correspondence between the original CT and US intensities. The method could not introduce the shadowing effect into the simulated images either. Daoud and Lacefield ${ }^{11}$ used a first-order $k$-space method and were able to simulate shadowing effects in their US 
images, but they had no real US images to compare with. Shams et al. ${ }^{12}$ and Kutter et al. ${ }^{13}$ also used the combination of reflection and transmission images and managed to capture the shadowing effect in the images by introducing use of contrast and edge enhanced images obtained from the CT images. But the drawback of their method was that it had no close relation to the physical processes of ultrasound backscattering.

In this work, a similar method using a combination of transmission and reflection properties to calculate the propagation of ultrasound was used. However, the mapping of the strength of the scatterers was different. The block diagram of this method is presented in Fig.4

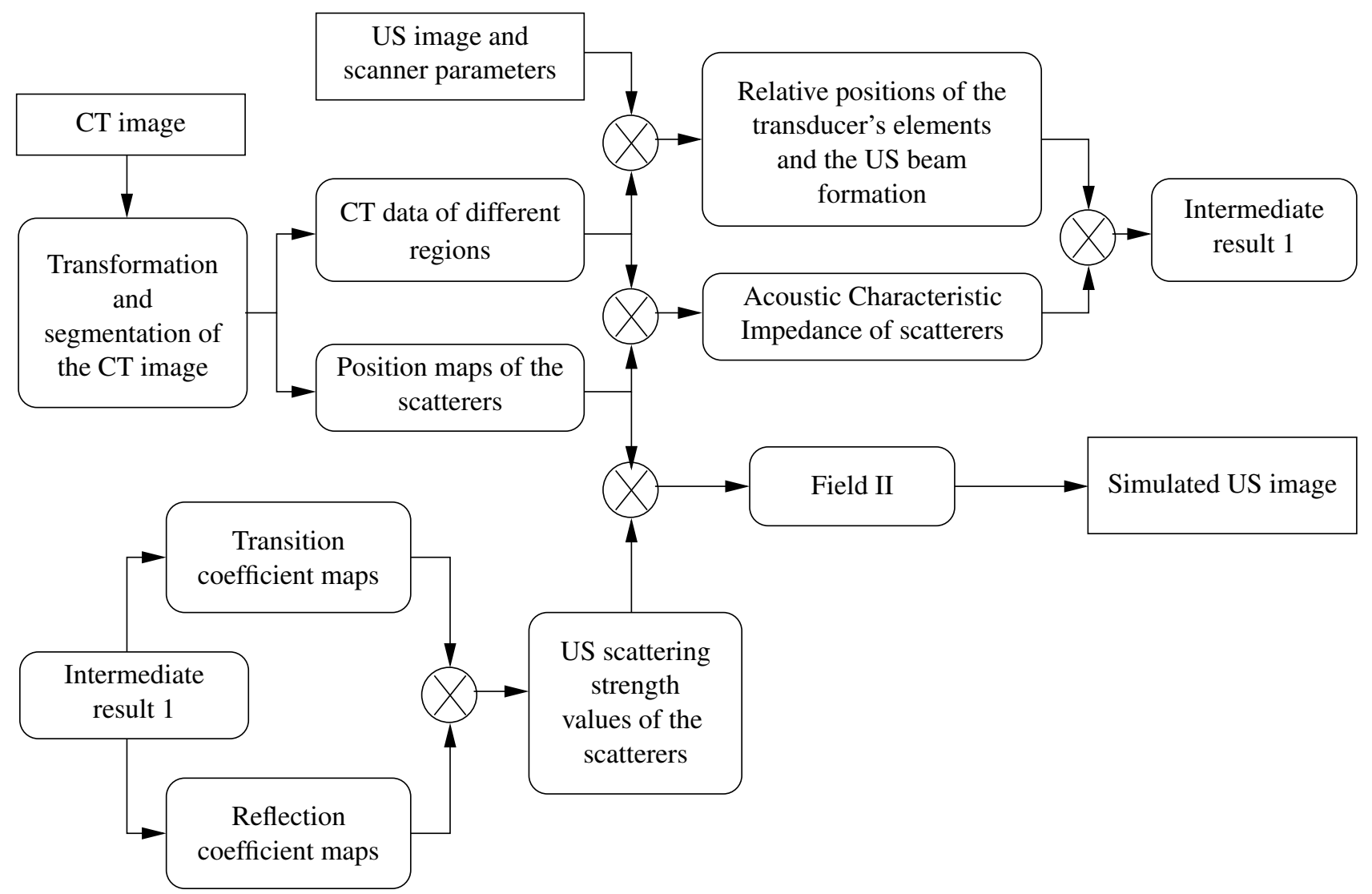

Figure 4. Block diagram of the US scattering strength distribution process

The CT images were cropped to get the same region of interest as in the US images. They were then segmented into regions containing fat, skin, soft tissue, and bone. Special regions were air-inside and air-outside the fish because the fish was CT scanned without surrounding water. In the CT image, the area outside the fish have Hounsfield Unit (HU) values of air, not water. But for the US scan, this region contains water. So for the simulation, the HU values of this region were then replaced by the HU values for water and a Gauss distributed scatterer strength and a uniform density distribution. The HU values of air-inside region remained the same, because this region also contained air in the US scan. The outputs at this stage were, thus, original CT data and position maps of the scatterers for the different regions. The combination of those outputs gave the Acoustic Characteristic Impedance (ACI) of the scatterers. Each region was assigned the values from local minimum to local maximum values, not a fixed value since the $\mathrm{HU}$ values of the points in that region were not the same. For instance, the minimum HU value in the bone region was 191, since the bone of a fish is softer than human bone; and the maximum HU value was 1200 . They were scaled to the ACI from $\left(3.6 \times 10^{6}\right)^{2}\left[M_{R a y l}^{2}\right]$ to $\left(7.38 \times 10^{6}\right)^{2}\left[M_{R a y l^{2}}\right]$, since they were intensity coefficients as in Table 1 .

The ACI of the scatterers were used to generate transition and reflection coefficient maps. The transition and reflection coefficient maps were created based on the fact that the energy of a US wave will change when it travels through a medium or from one medium to another. The transition coefficient maps the fraction of the energy remaining at each point in the 
image, when the acoustic wave travels through it. The shadow effect is quantified by the transition coefficient maps. The reflection coefficient maps the fraction of the energy being reflected at each point. It quantifies the edge effect or boundary effect seen in US images, where boundaries between different media are often enhanced by high brightness.

\begin{tabular}{ccc}
\multicolumn{3}{c}{ Table 1. HU to ACI conversion table } \\
\hline \hline Regions & HU range & ACI range $\left[\mathrm{MRayl}^{2}\right]$ \\
\hline Fat_skin & {$[-800,-190)$} & {$\left[1.5 \times 10^{6}, 1.5 \times 10^{6}\right)^{2}$} \\
Soft tissue & {$[-190,190)$} & {$\left[1.55 \times 10^{6}, 1.74 \times 10^{6}\right)^{2}$} \\
Bone & {$[190$, maximumvalue $]$} & {$\left[3.6 \times 10^{6}, 7.38 \times 10^{6}\right)^{2}$} \\
Water (replace air-outside) & $X$ & $\left(1.48 \times 10^{6}\right)^{2}$ \\
\hline
\end{tabular}

The transmission and reflection coefficient ${ }^{14}$ maps were given by

$$
\begin{gathered}
T_{n}=\prod_{k=1}^{m} T_{k} \\
T_{1}=1 \\
T_{k}=\frac{4 Z_{k} Z_{k-1} \cos ^{2} \theta_{i_{k}}}{\left(Z_{k} \cos \theta_{i_{k}}+Z_{k-1} \cos \theta_{t_{k}}\right)^{2}} \\
R_{n}=\left(\frac{Z_{n} \cos \theta_{i_{n}}-Z_{n-1} \cos \theta_{t_{n}}}{Z_{n} \cos \theta_{i_{n}}+Z_{n-1} \cos \theta_{t_{n}}}\right)^{2},
\end{gathered}
$$

where $\theta_{i}, \theta_{t}$ are angle of incidence and transmission. Assume that there were only normal incidence and transmission $\cos \theta_{i_{k}}=\cos \theta_{i_{n}}=\cos \theta_{t_{k}}=\cos \theta_{t_{n}}=1 . n$ is a point in the depth direction, $T$ is the transmission mask points, $R$ is the reflection mask points, $m$ is the number of points in depth direction from the transmit element, and $Z$ is the ACI of a point.

The US scattering strength maps of the scatterers are given by

$$
I M G_{H U U S}(x, y)=\alpha \cdot T(x, y) \cdot B(x, y) \cdot\left(1+e^{\beta * R(x, y)}\right)
$$

where $T(x, y)$ is the transmission coefficient maps; $B(x, y)$ is the ACI map; $R(x, y)$ is the reflection coefficient map; $\alpha, \beta$ are the shadowing effect coefficient and edge-enhancing coefficient, respectively. They are used to adjust the contrast of the simulated US images.

The relative position of the transducer elements and information on beam-forming algorithm of the scanner were obtained from the measured US images and the parameter files of the scanner. In this work, a 192 element transducer was used; the maximum number of active elements was 64 to beamform one line in the US image. The acoustic waves were beamformed with a fixed focus in transmit mode and dynamic focus in receive mode.

Finally, the Field II program was used to calculate the scattered sound field from a collection of sources defined by the scatterers in $I M G_{H U U S}(x, y)$. To achieve fully developed speckle pattern US image from many random scatterers, there should be 10 scatterers in a resolution cell. ${ }^{15}$ The resolution cell was calculated by

$$
\begin{gathered}
\text { rescell }=N \times \lambda \times F W H M_{l} \times F W H M_{a z i} \\
F W H M_{i}=F \#_{i} \times \lambda
\end{gathered}
$$

where rescell is the resolution cell, $N \times \lambda$ is the pulse length, $i$ is denote of $l$ and $a z i$ which stand for lateral and azimuth direction, respectively, and $\lambda$ is the wave length. The number of scatterers should be used in this simulation was calculated by

$$
n \text { scatt }=10 \times \frac{\text { imgdim }}{\text { rescell }}
$$

where $n s c a t t$ is the number of scatterers, imgdim is the dimension of the US image. In this simulation, the resolution cell were rescell $=0.1053 \mathrm{~mm}^{3}$, the image dimension were $i \mathrm{mgdim}=9680 \mathrm{~mm}^{3}$, and the number of scatterers was about 
$10^{6}$. Hence, each simulated US image was generated using $10^{6}$ point scatterers. The positions of the scatterers were within the position maps and were uniformly distributed. The amplitude values of the scatterers were the values of those scatterers in (5) and were random amplitude from a Gaussian distribution to model the difference in the density and speed of sound perturbations in the tissue. It took 5 to 6 hours to generate a $(38.9 \mathrm{~mm} \times 55.3 \mathrm{~mm})$ simulated US image of the fish with $10^{6}$ scatterers using 35 machines in the cluster at CFU.

\section{RESULTS}

The segmentation of the CT images of the fish is presented in Fig. 5. The 3D reconstruction from CT images of the fish is in Fig. 5a. A sample CT image of the fish was used for the simulation and is in Fig. 5b. The position of the CT image relative to the fish is at the white vertical line at $20 \mathrm{~mm}$ in the lateral direction in Fig. 5a. The position map of the different regions of the slice is Fig. 5c, where value 0 is for the air-outside region, value 1 is the fat-skin region, value 2 is the soft tissue region, value 3 is the bone region. Value 4 was assigned for air-inside region. But there was no air-inside region in this image, so there was only 3 types of values. The transmission coefficient map of the CT image is presented in Fig. 5d. It shows the shadow in the simulated image appeared. The reflection coefficient map is in Fig. 5e, and all the boundary area between different regions was captured.

The simulated US images for this section of the fish are presented in Fig. 6. In Fig. 6b, the pronounced shadowing effect of the US image was captured. The strongest shadows were due to the bones (most clearly seen in the center of the image) and the transition zones fish fin to water and water to fish body (upper left of the image). The edge-enhancing effect made the outline of the upper part of the fish which is close to the transducer, brighter than the one in the shadow in the lower part. The same phenomenon appears in the measured US image of the fish in Fig. 6a. The horizontally enhanced shadows in the concave zone at the lower part in the right side of the fish in Fig. 6a also appeared in Fig. 6b. The speckle pattern in the simulated US image appears much more uniform than in the measured image. One of the reasons is that the FWHM of the US imaging system is greater than the one simulated with Field II. The change in size as a function of depth of the point spread function appeared both in Fig. 6a\&b. The FWHM in lateral direction of the point spread function is proportional to the depth. It becomes larger for the scatterers faraway from the transducer.

Future improvements should focus on developing a method that can generate a type of coefficient maps that can capture both the shadowing and edge enhancement effects. It could be done by using different values assigned to the ACI of the different regions.

\section{CONCLUSION AND FUTURE PLANS}

This work is the first time that an experiment on fish is performed using a combination of US and CT to generate simulated US images. A new method to make the US backscattering map from CT data has been developed that can capture many of the properties of real US image. This shows the advantages of simulation of US images of the same fish from CT data with other transducers and setups without performing the real experiments with US imaging system. The simulated US images of the fish not only capture the shadowing effects, but also take into account the noise of the medium.

This work is one step in an ongoing work to investigate the structure of the acoustical properties of marine organisms with the goal of improving the possibilities for direct acoustic in-situ identification. The next step will be to model the scattering from a whole fish first at high frequencies, but then also for lower frequencies suitable for more long-range underwater use.

\section{ACKNOWLEDGMENT}

This work is sponsored by grant from the Globalization project of the Technical University of Danmark (DTU). Special thanks to Stina Bjørk Stenersen Hansen, fisheries technician at the DTU-Aqua in Charlottenlund for supplying the fish specimens. 
a)

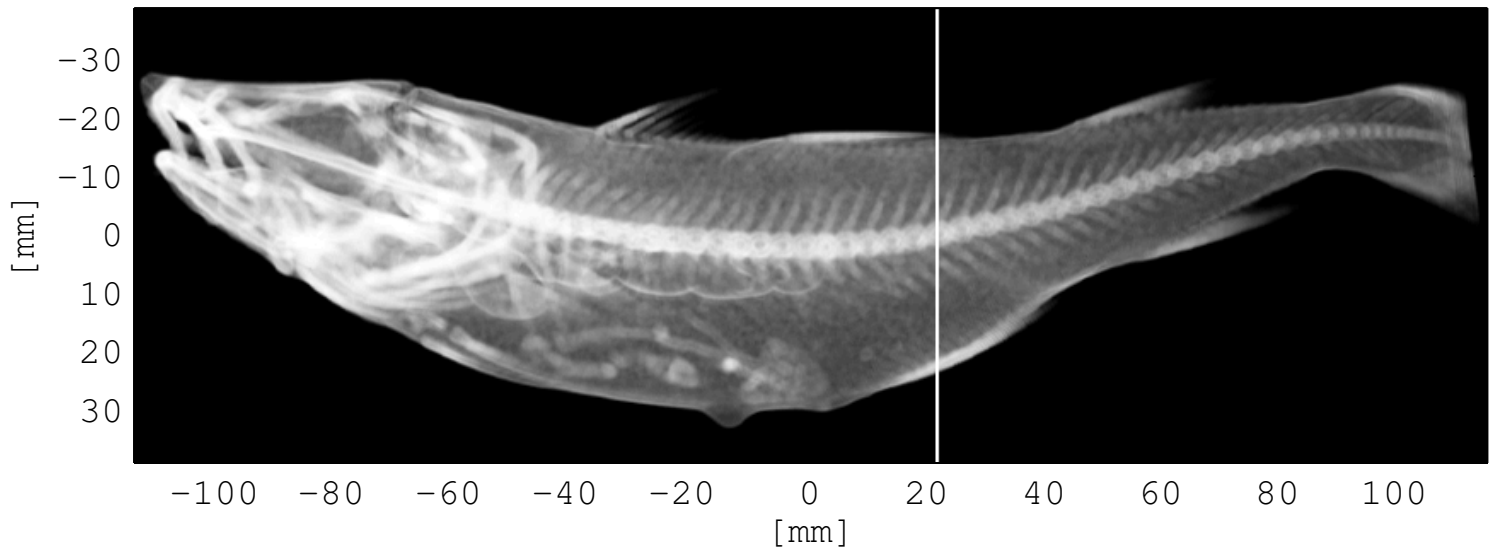

b) CT image of a slice

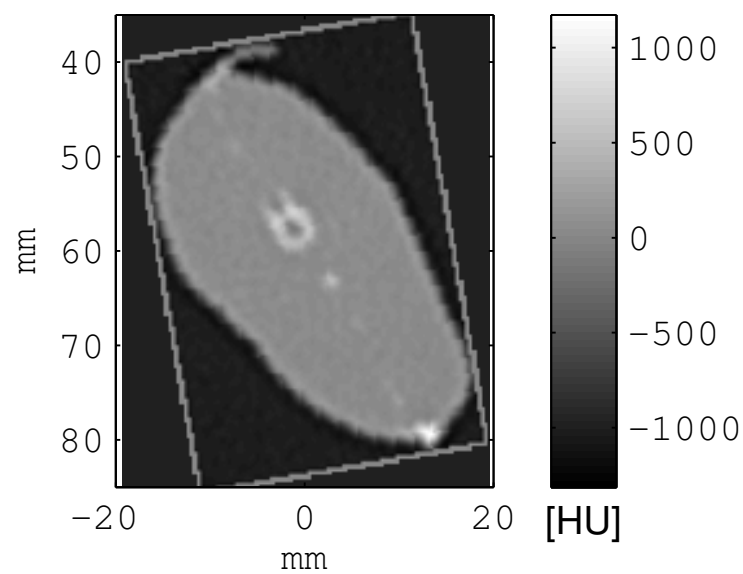

c) position map
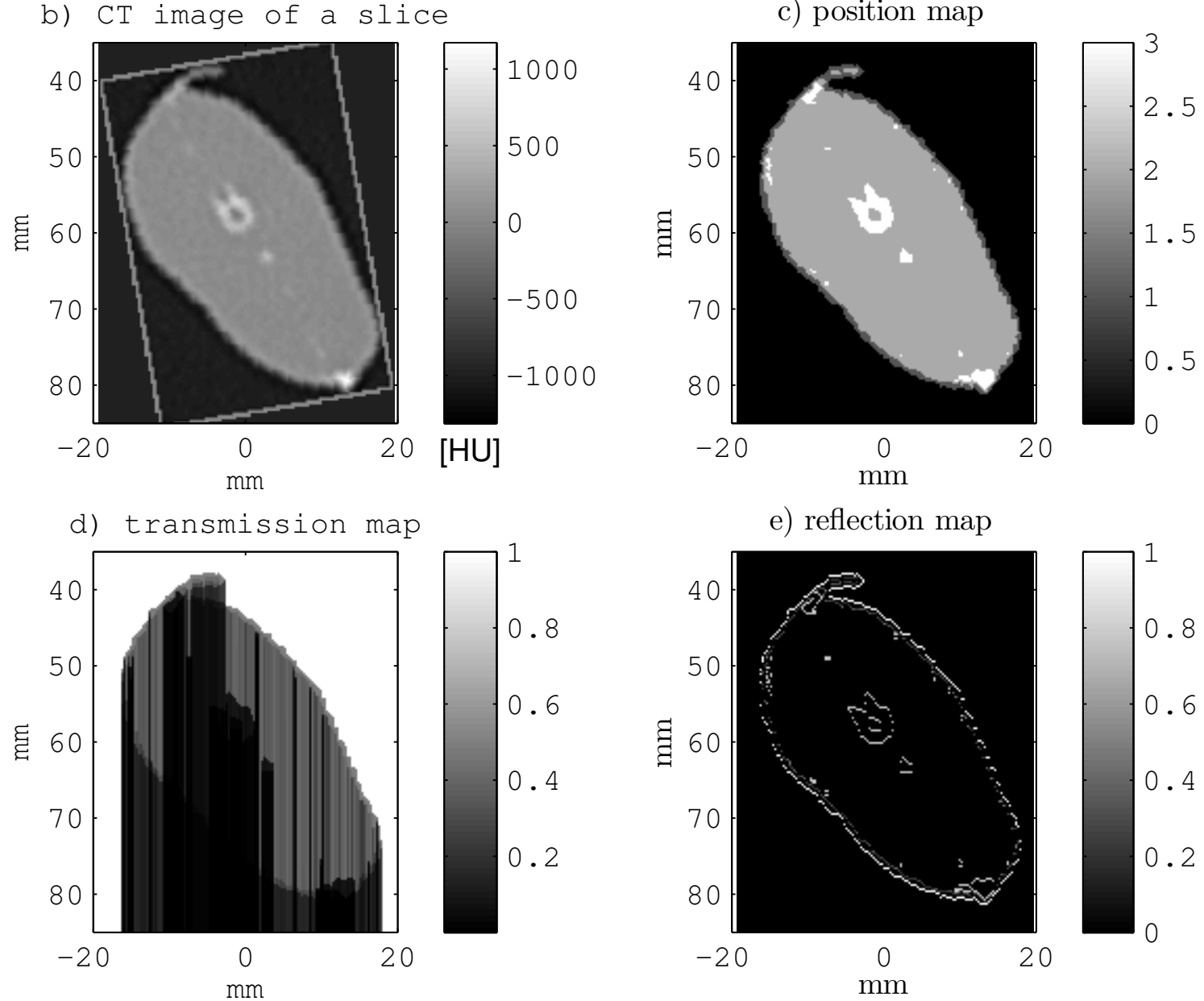

Figure 5. CT images segmentation and transformation; 3D CT image of the fish (a), CT image of a slice of the fish (b), position map of the different regions (c), transmission map (d), reflection map (e). 
a)

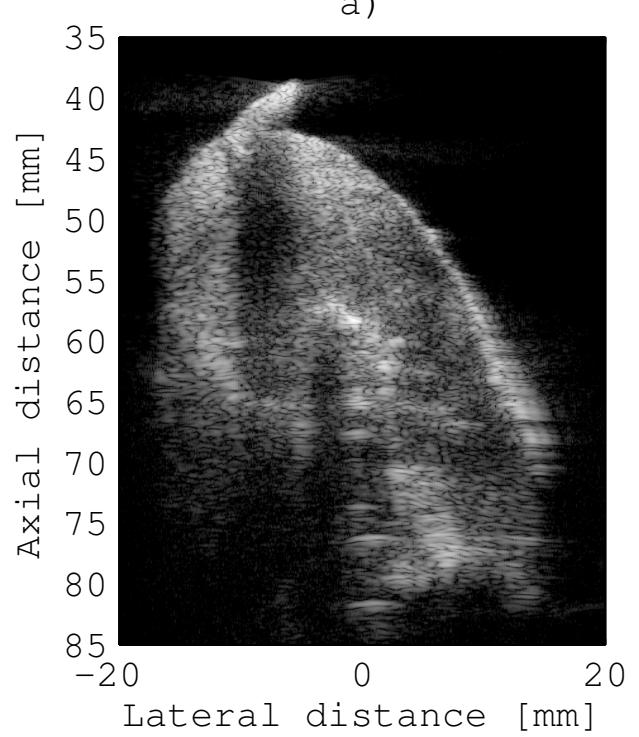

b)

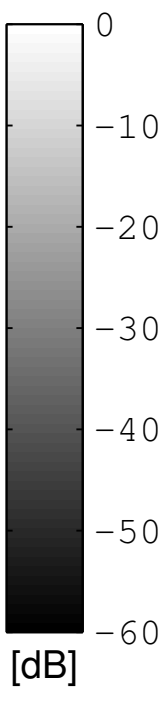

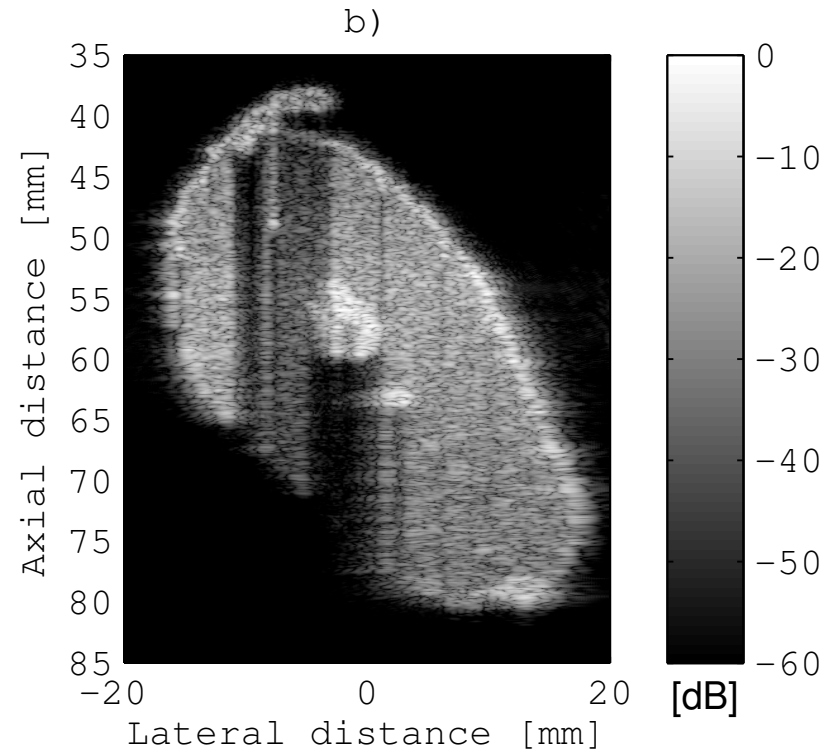

Figure 6. Measured US fish image (a), simulated US image of fish (b).

\section{REFERENCES}

[1] Anonymous. Report of the working group for international, pelagic surveys (wgips). In http://www.ices.dk/reports/SSGESST/2010/wgips10.pdf, volume 7629, jan 2010.

[2] J E Simmonds and D MacLennan. Fisheries Acoustics: Theory and Practice. Blackwell Science, second edition edition, 2005.

[3] R J Korneliussen, Y Heggelund, I K Eliassen, and G O Johansen. Acoustic species identification of schooling fish. ICES Journal of Marine Science, 66:1111-1118, 2009.

[4] B Lundgren and R N Nielsen. A method for the possible species discrimination of juvenile gadoids by broadbandwidth backscattering spectra vs. angle of incidence. ICES Journal of Marine Science, 65(4):581-593, 2008.

[5] J M Burgos and J K Horne. Acoustic characterization and classification of pelagic organism distributions. ICES Journal of Marine Science, 65:1235-1247, 2008.

[6] J A Jensen and N B Svendsen. Calculation of pressure fields from arbitrarily shaped, apodized, and excited ultrasound transducers. Ultrasonics, Ferroelectrics and Frequency Control, IEEE Transactions on, 39(2):262 -267, mar 1992.

[7] J. A. Jensen. Field: A program for simulating ultrasound systems. Medical and Biological Engineering and Computing, 10th Nordic-Baltic Conference on Biomedical Imaging, Vol. 4, Supplement 1, Part 1:351-353, 1996.

[8] M C Hemmsen, M M Petersen, S I Nikolov, M B Nielsen, and J A Jensen. Ultrasound image quality assessment: a framework for evaluation of clinical image quality. In Society of Photo-Optical Instrumentation Engineers (SPIE) Conference Series, volume 7629, March 2010.

[9] J L Dillenseger, S Laguitton, and E Delabrousse. Fast simulation of ultrasound images from a ct volume. Comput. Biol. Med., 39(2):180-186, 2009.

[10] W Wein, A Khamene, D A Clevert, O Kutter, and N Navab. Simulation and fully automatic multimodal registration of medical ultrasound. In MICCAI'07: Proceedings of the 10th international conference on Medical image computing and computer-assisted intervention, pages 136-143, 2007.

[11] M I Daoud and J C Lacefield. Distributed three-dimensional simulation of b-mode ultrasound imaging using a firstorder k-space method. Physics in Medicine and Biology, 54(17):5173-5192, sep 72009.

[12] R Shams, R Hartley, and N Navab. Real-time simulation of medical ultrasound from ct images. In D Metaxas, L Axel, G Fichtinger, and G Szekely, editors, Medical Image Computing and Computer-Assisted Intervention - MICCAI 2008, PT II, Proceedings, volume 5242 of Lecture notes in Computer Science, pages 734-741, 2008.

[13] O Kutter, R Shams, and N Navab. Visualization and gpu-accelerated simulation of medical ultrasound from ct images. Computer Methods and Programs in Biomedicine, 94(3):250-266, jun 2009. 
[14] J A Jensen. Estimation of Blood Velocities Using Ultrasound. A Signal Processing Approach. Cambridge University Press, New York, 1996.

[15] T A Tuthilla, R H Sperrya, and K J Parker. Deviations from rayleigh statistics in ultrasonic speckle. Ultrasonic Imaging, 10(2):81-89, apr 1988. 